NASA Technical Memorandum 103108

AIAA-90-2386

\title{
A Test Matrix Sequencer for Research Test Facility Automation
}

Timothy P. McCartney and Edward F. Emery

Lewis Research Center

Cleveland, Ohio

Prepared for the

26th Joint Propulsion Conference

cosponsored by the AIAA, SAE, ASME, and ASEE

Orlando, Florida, July 16-18, 1990

\section{NRSA}




\title{
A TEST MATRIX SEQUENCER FOR RESEARCH TEST FACILITY AUTOMATION
}

\author{
Timothy P. McCartney and Edward F. Emery \\ National Aeronautics and Space Administration \\ Lewis Research Center \\ Cleveland, Ohio 44135
}

\begin{abstract}
This paper describes the hardware and software configuration of a Test Matrix Sequencer, a general purpose test matrix profiler that was developed for research test facility automation at the NASA Lewis Research Center. The system provides set points to controllers and contact closures to data systems during the course of a test. The Test Matrix Sequencer consists of a microprocessor-controlled system which is operated from a personal computer.

The software program, which is the main element of the overall system is interactive and menu driven with pop up windows and help screens. Analog and digital input/output channels can be controlled from a personal computer using the software program.

The Test Matrix Sequencer provides more efficient use of aeronautics test facilities by automating repetitive tasks that were once done manually.
\end{abstract}

\section{Introduction}

In aeronautics research, test time in a research facility is a valuable and limited resource. Optimal facility utilization requires not only an increase in the number of test programs, but also in the amount of data acquired during any given test program. Any increase in utilization is greatly desired and the facility becomes a more productive resource.

Remote control of variables, such as model geometry, probe position, and facility valves, has been used for sometime at NASA Lewis to enhance productivity by reducing the time required to change these variables.

The next logical steps in the path toward increasing facility utilization are to minimize the time between data points and to maximize the quality of data taken at each test point. Automating the entire test matrix, which consists of a sequence of multiple test points, is a means of increasing productivity.

Previous test matrix automated systems at Lewis have required lengthy setup times, inflexible sequencing options, limited analog and digital input/ output options, and limited programmability. These earlier systems did not allow for on-line adjustments, operator intervention, or proficient configuration changes.

For these reasons NASA Lewis set out to develop a system that would provide flexibility and increased operational capability. The system developed, the Test Matrix Sequencer (TMS), is a general purpose, programmable, personal-computer-based set-point processor (fig. 1). The TMS consists of a microprocessor subsystem with analog and digital input/output channels controlled from a personal computer by a menu

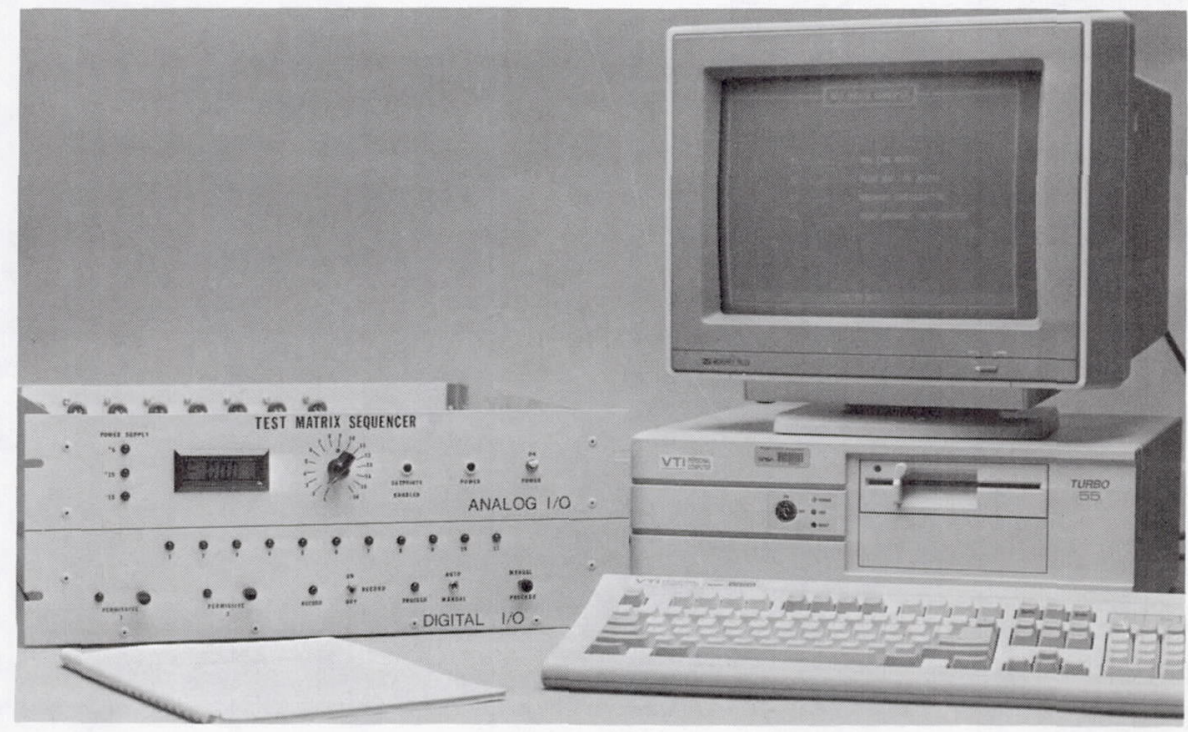

Fig. 1 Test Matrix Sequencer system. 
driven program. Below is a list of the system's major features:

- 14 Analog I/O

- 14 Facility system analog inputs

- 15 Discrete I/O

- Variable ramping of outputs

- 0.1 Percent accuracy

- 50 Unique test points

- Fully programmable

- Multiple test sequences

- Menu driven

- Help screens

- Interactive checkout

- File handling

- Simple operator panel

- Ease of operation

- Serial communications

- Data recording control

The microprocessor subsystem handles all of the low level I/O in a parallel processing fashion. This allows the personal computer to continue to run the test matrix, monitor the channels, and keep the operator informed of the test status.

The software program, which is the heart of the system, allows the user to configure, store, and run complete test matrices as well as to directly control the I/O channels from the keyboard. The system also, at each test point, verifies controller operation, turns on recorders, waits for user permissives, and handles the data system communications. Figure 2 shows how the system can be integrated in an automated test facility.

The TMS has proven to be ideal for use in the aeropropulsion research test facilities at Lewis since it automates repetitive tasks such as the positioning of actuators for multiple test conditions. The TMS accomplishes these tasks with far more accuracy, repeatability, and speed than manual operation.

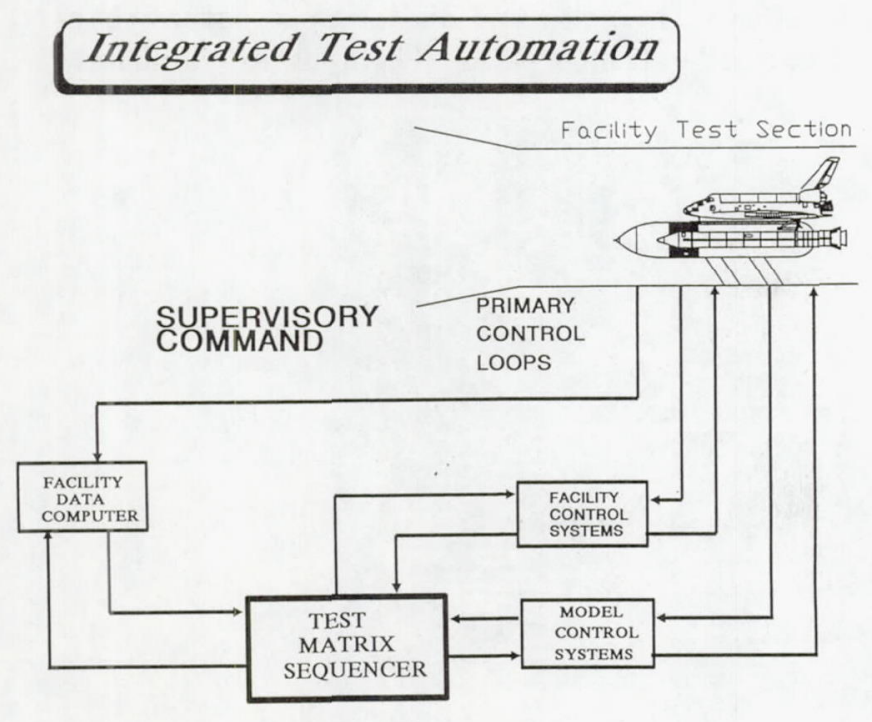

Fig. 2 Test Matrix Sequencer in an automated test facility.

\section{System Overview}

The TMS (fig. 3) consists of an electronic hardware system with 14 analog and 15 digital user configurable I/O channels and a software program running on a personal computer. The system can acquire $\mathbf{1 4}$ additional analog inputs from the facility data system. The program is written in Turbo Pascal and is a fully integrated, menu-driven system.

The electronic hardware, consisting of two microprocessors with digital and analog boards, is housed in a 7-in.-high rack-mountable chassis with an active front panel. The analog and digital boards each consists of a microprocessor with a passive $\mathrm{I} / \mathrm{O}$ rack that will accept optically isolated $\mathrm{I} / \mathrm{O}$ modules. The modules contain all the signal conditioning required to interface with field devices and the onboard microprocessor. Analog modules provide 12 -bit resolution in a variety of full-scale ranges for both the outputs and inputs. Digital modules are used for sensing on/off voltages in a wide range of dc voltages as well as dry contact closures.

The Test Matrix Sequencer Software consists of four major routines:

- Run Time Matrix

- Print Run Time Matrix

- Hardware Configuration

- Print Hardware Configuration

The Run Time Matrix routine allows the user to define, load, save and run the test matrices. The Hardware Configuration routine enables the user to define the analog and digital I/O channel parameters for the run time portion of the program and perform file operations. The Print Hardware Configuration and Print Run Time Matrix routines provide printouts of the user-defined sequences and configurations.

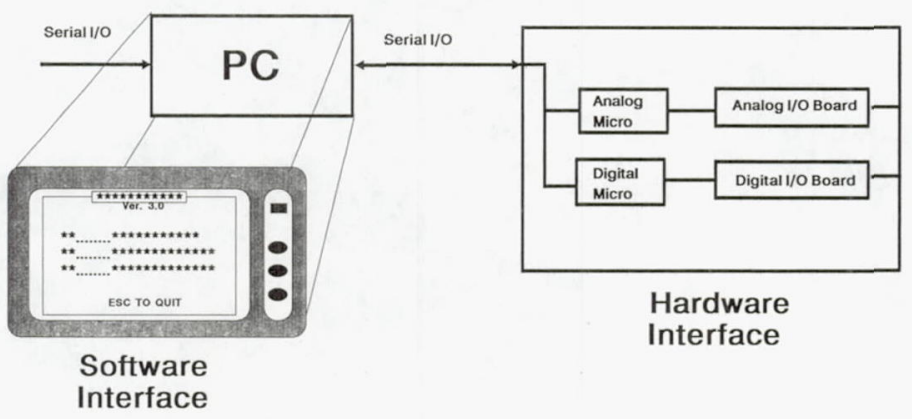

Fig. 3 Test Matrix Sequencer block diagram. 


\section{Hardware}

The Test Matrix Sequencer electronics are contained in one 7 -in.-high, rack-mountable chassis. The front panel (fig. 4), which is divided into two sections, displays the status of the Test Matrix Sequencer independent of the software program. The autoranging digital voltmeter can be used to monitor any of the analog channels as well as to test the power supplies. A light-emitting diode on each digital I/O channel indicates the channel status. Other front panel controls include manual overrides for the system input channels and a switch to enable or disable data recording system sequencing.

Internally, the system consists of analog and digital I/O boards, power supplies, and a switch panel (fig. 5). Each I/O board contains a microprocessor and a passive mounting rack.

Each microprocessor performs the following functions: serial communication with the personal computer, onboard diagnostics, error checking, and low-level communication to I/O channels. The microprocessor communicates with the host computer via an RS 422 / 485 bus operating at $19.2 \mathrm{~K}$ baud. The RS $422 / 485$ card, which plugs into a slot in the personal computer, protects the system from transients and communicates serially with electronic hardware up to $5000 \mathrm{ft}$ away. The $19.2 \mathrm{~K}$ baud rate was selected because it can provide maximum throughput communication from a personal computer to the I/O modules.

The passive mounting racks accept commercially available I/O modules. These racks allow the placement of modules in any position and allow for the replacement of modules without disturbing field wiring.

The analog and digital I/O modules are of a general-purpose, optically isolated plug-in type that are controlled by a personal computer through the local microprocessor.

The digital input modules are capable of detecting voltages from 2.5 to $28 \mathrm{~V}$ dc and feature input filtering, high noise rejection, and transient-free switching. Two types of digital output modules are available: form A mechanical relays and voltage outputs. The

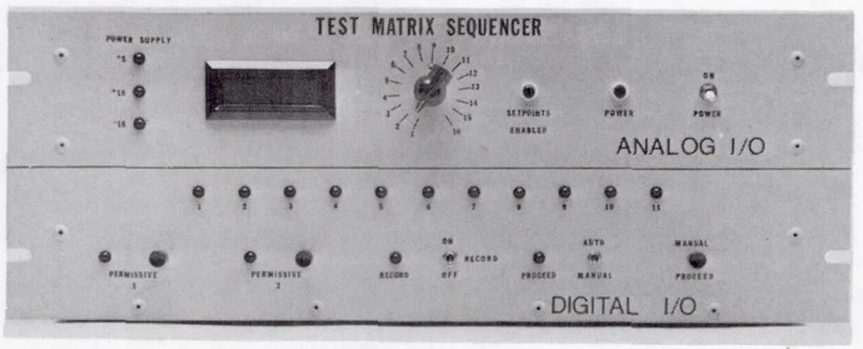

Fig. 4 Test Matrix Sequencer front panel.

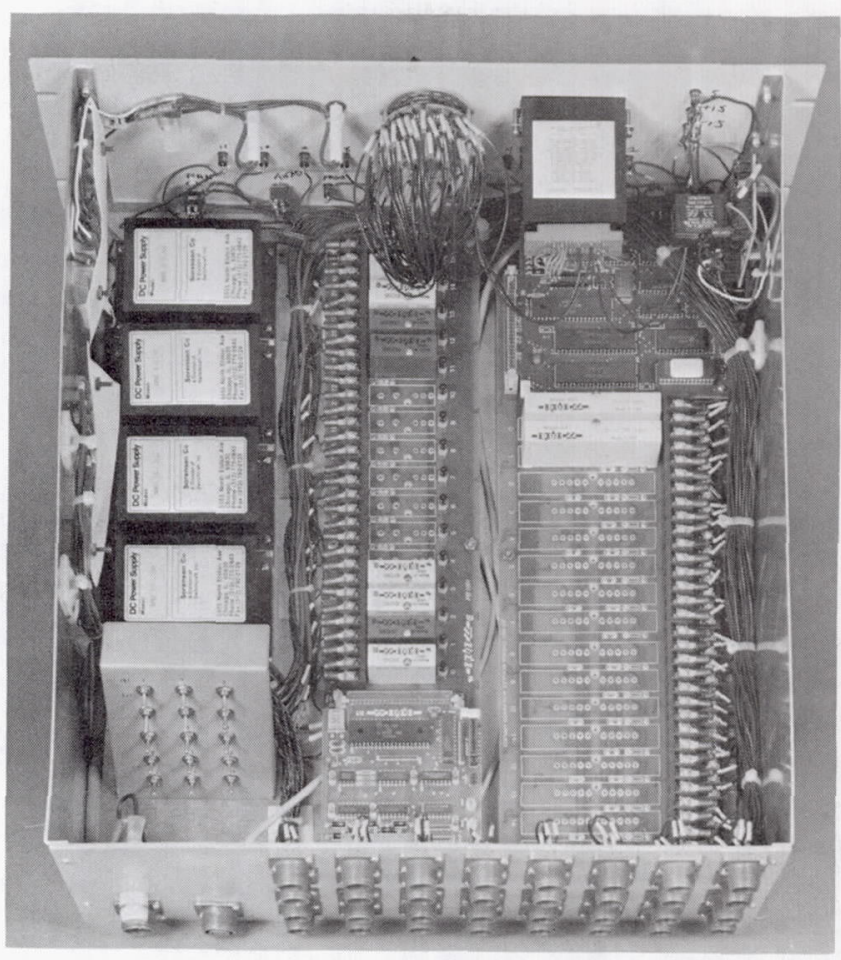

Fig. 5 Test Matrix Sequencer internal view.

form A mechanical relays furnish a dry contact closure, and the voltage output modules control and switch voltages from 5 to $60 \mathrm{~V}$ dc.

The analog I/O modules are available in four voltage ranges ( 0 to 5,0 to $10, \pm 5, \pm 10 \mathrm{~V} \mathrm{dc}$ ) with 12 -bit resolution and accuracy of \pm 0.1 percent of span. The analog input modules have differential inputs, and all I/O modules are optically isolated from the field devices. Field connections to the TMS are made through the two-pin connectors on the back of the chassis (fig. 6).

\section{Software}

The software program that operates the TMS is menu driven with windows and fully integrated help screens. All options in the program may be selected

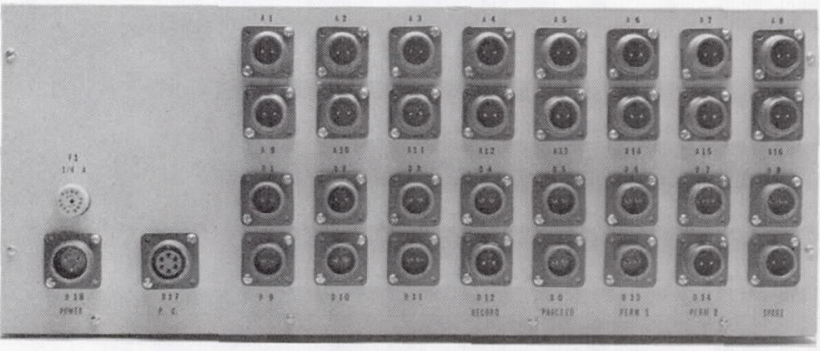

Fig. 6 Test Matrix Sequencer rear panel interconnect. 
from a moving bar type menu or from the function keys. The program consists of four major routines: the Run Time Matrix, Print Run Time Matrix, Hardware Configuration, and Print Hardware Configuration routines. Each routine is selected from the main menu (fig. 7).

The Run Time Matrix routine allows the user to create, test, and run test matrices. It also allows manual operation of the inputs and outputs for checkout purposes. When the user runs this routine, a hardware configuration file must be specified. The configuration file is created with the Hardware Configuration routine and provides all the necessary channel information to run TMS.

The Print Run Time Matrix routine provides the user with a hard copy of the user-defined test matrix.

The third option on the main menu, the Hardware Configuration routine, allows the user to identify the type of hardware to be used, the symbolic names for channels, conversion rules, and other information specific to the I/O channel. Once the Hardware Configuration has been set up, it can be saved on disk and used to build a number of test matrices.

The Print Hardware Configuration routine is the last option and provides a hard copy record of the specific hardware configuration.

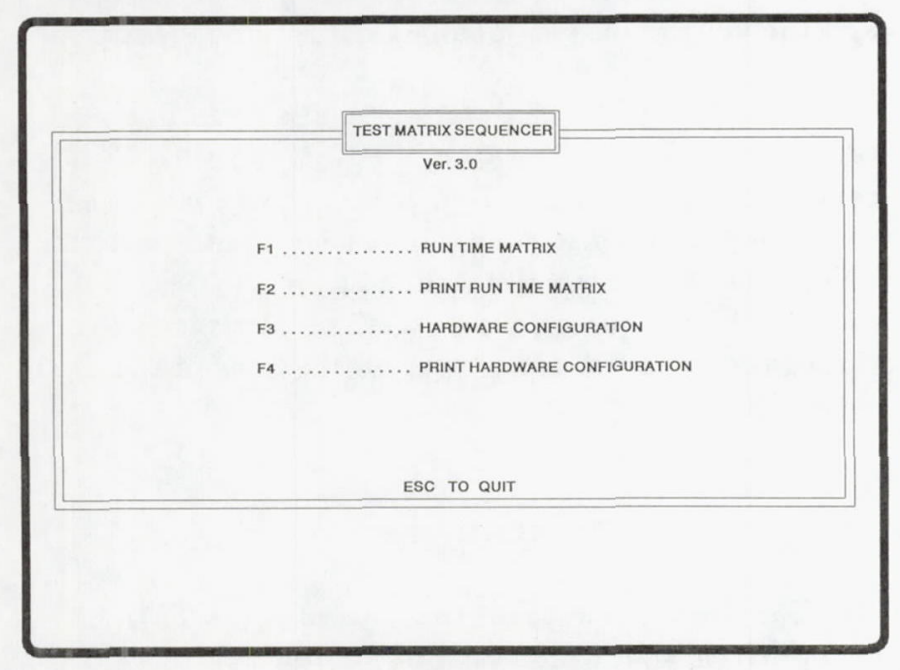

Fig. 7 Main menu.

\section{Run Time Matrix}

The Run Time Matrix portion of the program is where the user actually creates and runs the test matrix. The test matrix is built from multiple test points or subsequences. Each test point consists of the following steps :

- Output analog set points

- Verify analog inputs

- Check system permissives

- Set discrete and digital outputs
- Check discrete and digital inputs

- Start countdown timer

- Close data system contact

- Wait for data system contact

Each test point in the test matrix can use some or all of these options listed.

When the Run Time Matrix option is selected from the main menu, the user is prompted for a hardware configuration file name. The hardware configuration file contains all of the necessary channel information.

The main screen of the Run Time Matrix routine (fig. 8) is in a spreadsheet type of format, with the analog channels on the left and the test points across the top. Each analog output channel set up in the Hardware Configuration routine is displayed on this page along with its associated labels and limits. All the set points are defined on the Analog Output Page. Each set point is entered directly in the spreadsheet by moving the cursor to the desired location and entering the new data.

Set points may also be entered dynamically into the test matrix through the asynchronous communications channel. This enables the set points to be assigned to the test matrix while the sequencer is running. The facility data system provides the set points on the serial communication channel, and the TMS acquires the set points at run time. With this capability the set points can be a function of model conditions during a run.

An optional ramp time may be entered with each set point on the Analog Output Page. This enables the user to ramp an analog output from one test point to the next. Ramp durations can extend from $1 \mathrm{msec}$ to several minutes. The last column on the Analog Output Page lists all ramp times for the test point defined by the cursor position in the spreadsheet.

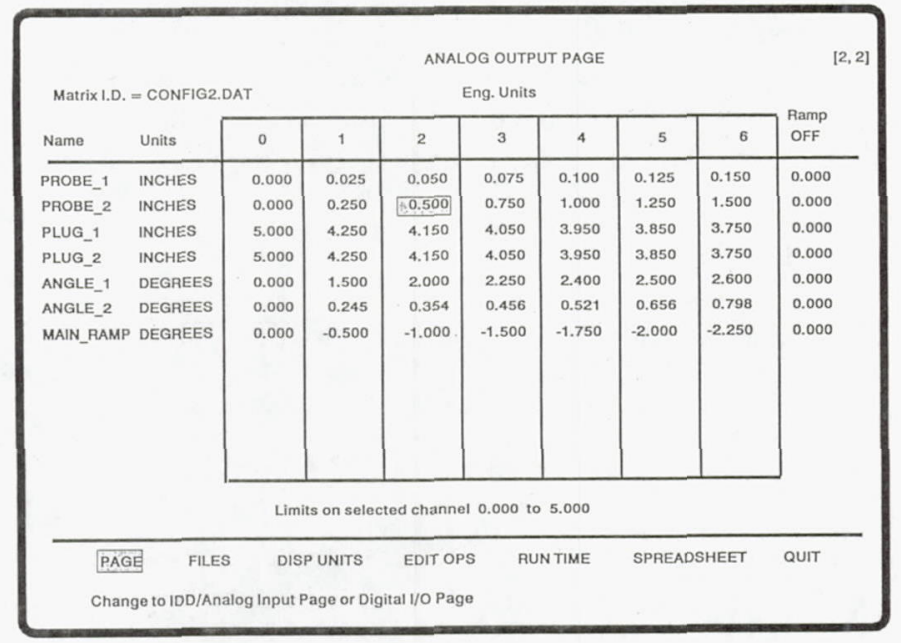

Fig. 8 Run Time Matrix Routine - analog output page. 
The software limits for the current channel are displayed below the spreadsheet. These limits are defined by the user and restrict the range of output values entered in the test matrix.

Each test point has associated with it an Analog Input Page and Digital I/O Page in addition to the information on the Analog Output Page. The Analog Input Page is used to define the analog input for the test point as well as to set up the system permissives and countdown timer for the test point. The Digital Input Page is used to define the discrete or digital input and output conditions for the test point.

On the Analog Input page (fig. 9) an input range or tolerance may be specified for each input channel. These ranges are relative to the value set on the Analog Output Page for the associated output channel.

There are two methods for inputting analog data: through input modules on the analog $\mathrm{I} / \mathrm{O}$ board and through an asynchronous communications channel (RS 232). The communications channel inputs data from control room readouts called individual digital displays (IDD's). The IDD's are controlled by the facility data system via a daisy chain communication network in the control room. This gives the TMS the potential for an additional 14 analog inputs.

Other options on the Analog Input page are the setting of the system Input Permissives and the test point Wait Time. The input permissives are two input contacts that can be checked after the analog inputs are verified during the run sequence.

The Digital I/O page (fig. 10), also associated with each test point, allows the user to define the digital output and input conditions. The outputs defined in the hardware configuration program are listed on the left, and the inputs on the right. Displayed with the digital outputs are the initial conditions for each channel. The options for the output channels are

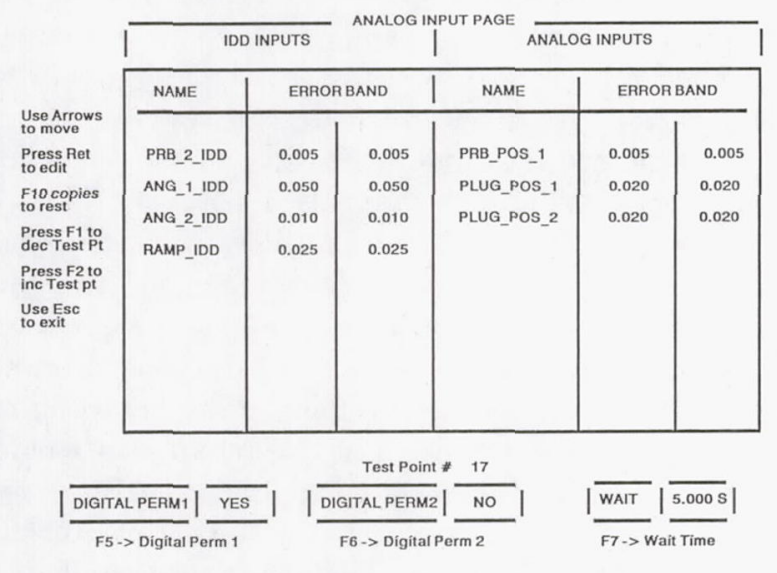

Fig. 9 Run Time Matrix Routine - analog input page.

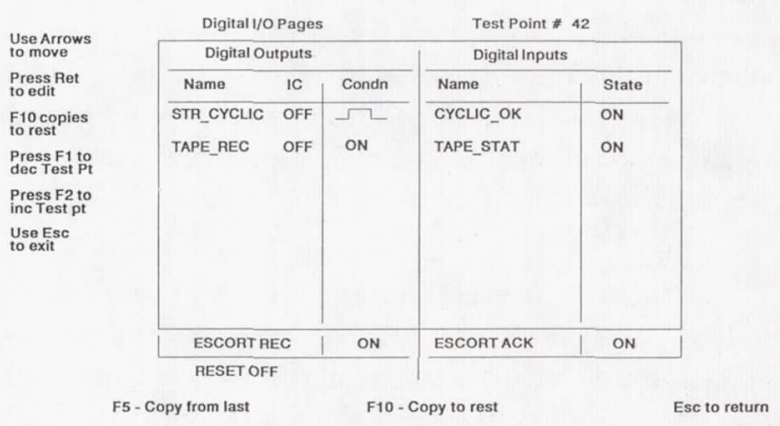

Fig. 10 Run Time Matrix Routine - digital I/O page.

on, off, pulse on, pulse off, and don't care. Each output can be reset to the initial conditions after each test point or left in the previously set state. The input channels can be set to wait for either an on, off, or don't care condition. The data system record and acknowledge contacts may be set on the bottom of the page. These contacts initiate the data acquisition and wait for its completion.

The conditions defined on the Analog and Digital Pages are unique to the test point that is displayed at the top of the page. Each Page may be either configured individually or copied from other test points. This gives the user maximum flexibility when creating the test matrix.

Once the matrix is defined, the user can save it to disk and either run it or build another matrix based on the same hardware configuration. This way there can be multiple test matrices built and ready to run on disk.

Once the test matrix has been created, the user must define the run sequences. The program allows four sequences (main, A, B, and C) to be stored at any one time. Each sequence can be used individually or in combination with other sequences to form loops. The TMS may be configured to have the main sequence call one of the lower order sequences such as $A$, $\mathrm{B}$, or $\mathrm{C}$. In addition sequence A may call B, and B may call $C$. This ability to call multiple subsequences gives the user four levels of looping when defining a test sequence. Each of these sequences is defined with a sequence editor. The validity of each sequence is verified before it is stored.

Before the TMS can actually be operated, it must be brought on-line. This involves turning on the ana$\log$ outputs. This is a software-controlled switch that ensures that all the outputs are at a safe value as defined by the home position (test point 0 ). With the 
outputs active or $\mathrm{ON}$ the user may run and control the inputs and outputs. With the outputs inactive the TMS runs in a simulation mode. This mode of operation runs the same sequences as the normal operation except the $\mathrm{I} / \mathrm{O}$ are disabled.

The TMS software provides three ways of modifying the analog and digital I/O. The three modes are

- Direct interactive

- Single test point

- Normal sequence

The direct interactive mode allows the user to modify, in engineering units, any output channel from the keyboard and to monitor its associated input channel. This mode is useful in the initial setup and in the calibration of analog $\mathrm{I} / \mathrm{O}$ channels. The original and current set points are displayed along with continuously updated input values. The TMS software will display the effects of changing the outputs in real time.

In single test point mode, the TMS software runs an individual test point. This mode is also useful in the setup and checkout phase of a test. A single test point can be checked independently from the rest of the matrix. This affords the user the ability to examine how modifying multiple set points affects the test model.

In the normal sequence mode the program executes the test points in the sequence previously defined. It is in this mode that the Run Time Screen is displayed to keep the user informed of the status of the run.

The Run Time Screen (fig. 11) informs the user of the status of each item by the use of screen attributes. The attributes are reverse video for inactive, blinking for active, and normal video for completed items. On completion of the run, the display returns to the analog output page ready for another run or editing.

During the operation the user may stop or abort the run. Stopping the run simply causes execution to

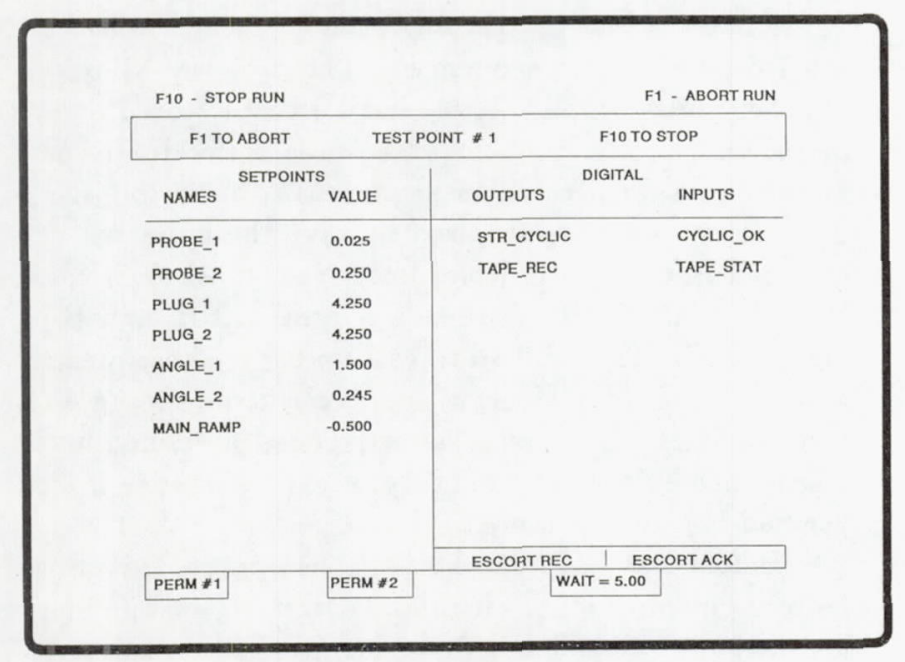

Fig. 11 Run Time Matrix Routine - run time screen. stop at the current point and returns to the analog output page. Aborting causes the execution to stop and sets all the outputs to the home position (test point 0).

Other features of the Run Time Matrix portion of the TMS software are full featured editing including insertion and deletion of full or partial test points, multilevel integrated help screens, and operation by either a menu selection or function keys.

\section{Print Run Time Matrix}

The Print Run Time Matrix routine prints the test matrix in one of two formats: test point or input/output. Both formats prompt the user for a file name and display a directory listing of the available matrix files.

The Test Point Format prints all the information for each test point specified.

The I/O Format prints the test matrix information in the following order: analog outputs, analog inputs, digital permissives, digital outputs, and digital inputs. The user is prompted for the range of test points to be printed.

\section{Hardware Configuration}

The Hardware Configuration routine selected from the main menu is used to identify all the necessary information for the Run Time Matrix portion of the system. When this option is selected, the main configuration screen is displayed identifying the 14 analog and 11 discrete user I/O channels. (Four dedicated discrete I/O channels are available in Run Time Matrix Routine.) An existing configuration file may be loaded or a new one created from this screen. When an existing file is loaded, the user specified name of each channel is displayed adjacent to the associated channel number (fig. 12). These channel numbers are the physical slot numbers on the analog/digital I/O boards. Channels are selected by using the arrow keys to move the reverse video block to the desired slot.

A window pops up on the right side of the screen when one of the analog channels is selected. This window (fig. 13) identifies and allows editing of the configuration associated with this channel. The analog edit window contains the symbolic names associated with the channel, the type of $\mathrm{I} / \mathrm{O}$ it is, the conversion information, and the limit setting. The following paragraphs describe the fields in the analog edit window.

The first field in the analog window defines the symbolic name to be associated with that channel throughout the program. The next field tells the program whether the user has installed an analog input or output module. If an analog output is 


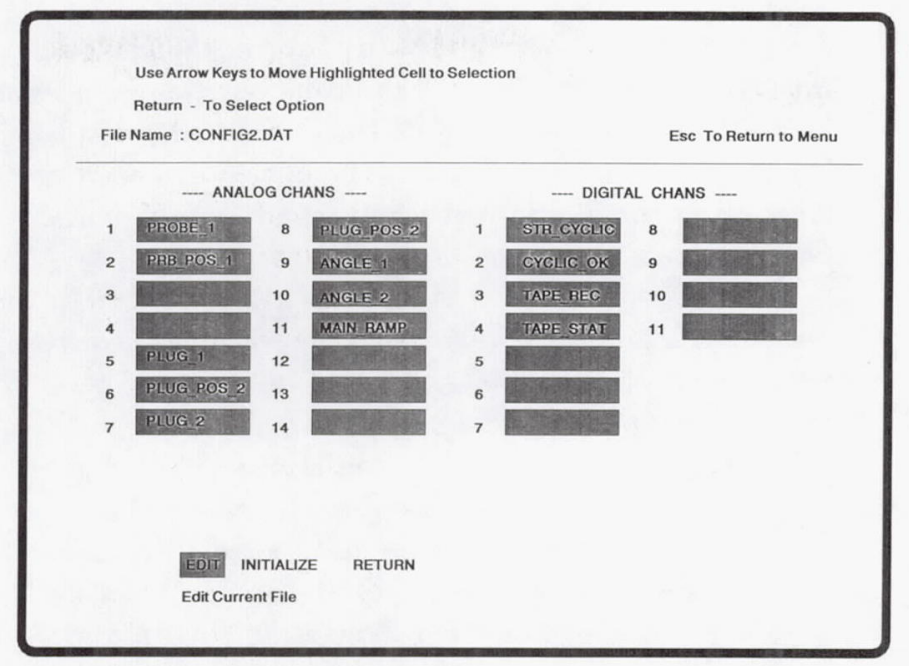

Fig. 12 Hardware Configuration Routine - main screen.

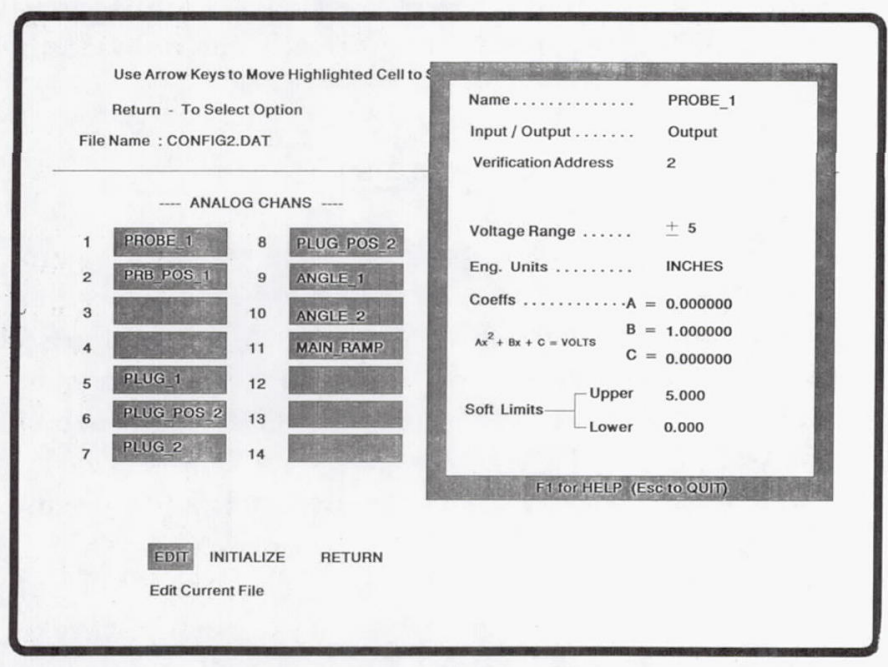

Fig. 13 Hardware Configuration Routine - analog $\mathrm{I} / \mathrm{O}$ screen.

selected, the Verification Address field is displayed. This address, which is an analog slot number or IDD address, specifies the channel or IDD that the sequencer will use to verify any actions taken by the current output channel. This is how the input channels described above are set up to monitor output channels.

The voltage range field identifies for the program the voltage range of the $\mathrm{I} / \mathrm{O}$ module installed. The engineering units label is an optional user specified name that will be displayed with the channel throughout the TMS software.

Another field in the analog edit's window is the conversion rule setup for both input and output channels. This is accomplished by correctly setting the coefficients parameters during the analog configuration. The coefficients supplied are fit to a quadratic equa- tion. This enables the TMS software to set the outputs and display the inputs in engineering units.

The last two fields in the analog window are the soft limits. These may be assigned values to ensure that the outputs remain within some predefined bounds. These limits are entered in engineering units.

Similar to the analog case, the selection of a digital channel causes a window to pop up on the left side of the screen (fig. 14). The options associated with the selected channel are displayed. The name is a label used to identify the channel throughout the TMS software as it was in the analog case. The second field simply identifies for the program whether the channel is an input or an output. If the channel is configured as an output, the third option allows the user to specify an initial condition (on/off).

The hardware configuration can be saved to a file on disk for use by the Run Time Matrix program once the configuration is complete.

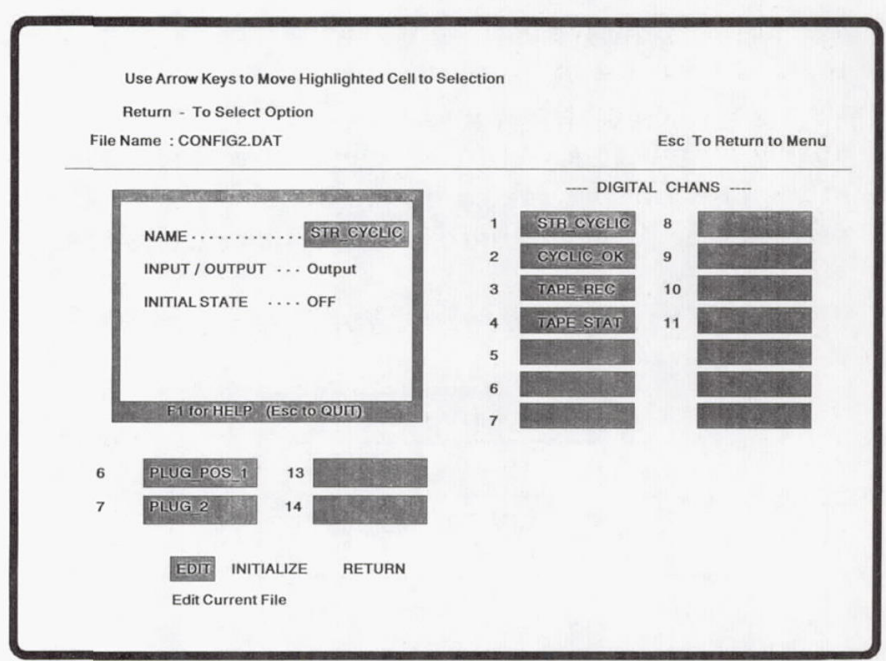

Fig. 14 Hardware Configuration Routine - digital I/O screen.

\section{Print Hardware Configuration}

This routine, selected from the main menu, provides the user with a hard copy of a specified hardware configuration. When selected, this routine displays a directory of all configuration files. The printed copy lists all the channels configured starting with the analog $\mathrm{I} / \mathrm{O}$ channels followed by the digital $\mathrm{I} / \mathrm{O}$ channels. 


\section{Applications}

The TMS has been used in several Lewis aeropropulsion research facilities and test programs. A general applications block diagram is shown in figure 15. Some of these include the Advanced Inlet Test in the 9 by 15 Foot Low Speed Wind Tunnel, the Counterrotating Advanced Turboprop Test in the 8 by 6 Foot Supersonic Wind Tunnel, and the Mach 5 Inlet Test in the 10 by 10 Foot Supersonic Wind Tunnel.

In its first application the TMS was used to position two bleed valves in the Advanced Inlet Test. In this test the TMS was configured with a pair of analog outputs used as set points to electrohydraulic servocontrollers. These controllers positioned the variable bleed valves. The TMS also had two analog inputs set up to read the position of the bleed valves to verify the controller's operation. In addition, the TMS was configured to take advantage of the permissive inputs to verify that the model was aerodynamically at the desired condition before data were taken. The TMS then provided the contact closure to the data system and waited for acknowledgement from the data system before proceeding to the next test point.

In the Counterrotating Advanced Turboprop Test, the TMS was set up to position a wake survey probe through a 28-point test matrix. The TMS provided the analog output to a controller and verified the position of the probe before taking data. Once the position was verified, the TMS provided a contact closure to the

\section{TMS Application Block Diagram}

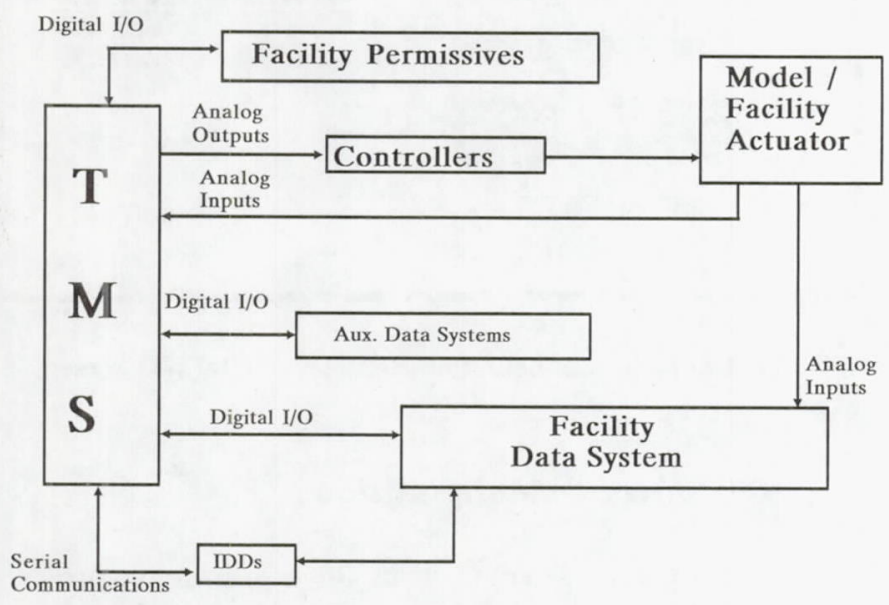

Fig. 15 General application block diagram. data recording system and waited for acknowledgement before continuing. If the probe position could not be verified, the TMS notified the operator by displaying an error screen to prompt the operator for action. The use of the TMS greatly improved the efficiency and repeatability of the probe surveys.

The TMS was used in the Mach 5 Inlet Test to position 12 probes in various configurations through a sequence of 25 test points. In this test, several test matrices were set up and saved on disk. Since each test matrix specified a probe configuration, configuration changes required only loading a new data file. The TMS fully automated the data acquisition by moving the probes to the required position, waiting for the desired model condition, triggering the data system, waiting for acknowledgment, and continuing to the next test point.

Automating the process saved valuable tunnel time in that the probes were positioned simultaneously instead of manual positioning of each one individually.

\section{Summary}

Although test automation is not new in the aeropropulsion facilities at NASA Lewis, never before has it been as easy or flexible. The Test Matrix Sequencer is a stand alone system dedicated to facility automation and increased productivity at the Lewis Research Center. The TMS has been used very successfully in three of the Center's major wind tunnels and, because of it's success, is targeted for use in several other aeropropulsion facilities. In conclusion, the TMS has proven to be a valuable tool during the setup, checkout, and running of tests at Lewis as well as providing for greatly improved facility utilization.

\section{Bibliography}

Turbo Pascal: Owner's Handbook (IBM version 4.0), Borland International, Scotts Valley, CA, 1987. Optoware Digital/Analog Driver for IBM PC (User Manual Version 3.0), Opto 22, Huntington Beach, CA. Sept. 1986.

Optomux: Analog User's Manual (3rd ed.), Opto 22, Huntington Beach CA, 1984.

Optomux: Digital User's Manual (3rd ed.), Opto 22, Huntington Beach, CA, 1984. 


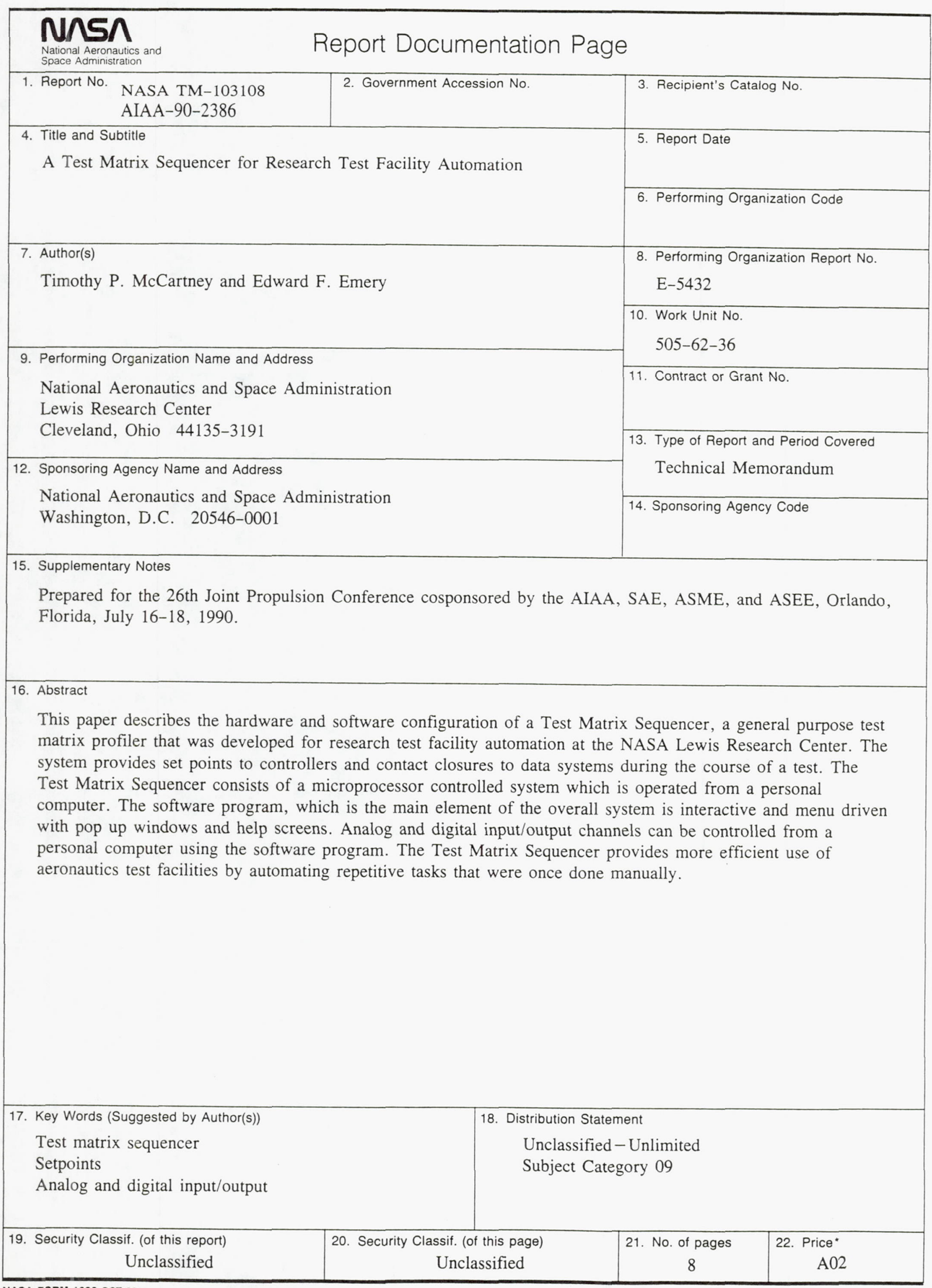

